\title{
La adición de Enterococcus faecium aumenta la respuesta inmune intestinal en cerdos en crecimiento
}

\author{
Herrera, F.V. ${ }^{1} ;$; Ciro, J. ' y Parra, J. ${ }^{1}$
}

'Grupo Biodiversidad y Genética Molecular BIOGEM. Departamento de Producción Animal. Facultad de Ciencias Agrarias. Universidad Nacional de Colombia. Medellín. Colombia.

\section{Palabras Clave adicionales}

Destete.

Lechones.

Probióticos.

Sistema inmune.

\section{RESUMEN}

El destete produce un periodo breve de ayuno y adaptación a una nueva ración sólida, la cual provoca disminución de la supervivencia de los lechones y proliferación de la microbiota patógena. Se ha propuesto la utilización de probióticos, evitando que los seres humanos consuman alimentados tratados con antibióticos. El objetivo de este trabajo fue determinar el efecto de la adición de cepas de probióticos en cerdos en crecimiento sobre las poblaciones celulares del sistema inmune (eosinófilos, basófilos, neutrófilos, monocitos y linfocitos) en intestino delgado. Se sacrificaron 35 lechones escalonadamente los días 1 (21 días de edad), 15 y 30 posdetete, y se extrajo completamente el intestino. Los animales fueron alimentados con dos dietas: dieta comercial con y sin la adición de antibiótico; a esta última se adicionaron los diferentes probióticos (Lactobacillus casei, Lactobacillus acidophilus o Enterococcus faecium) en el agua de bebida. Se utilizó un diseño de bloques al azar en un arreglo de parcelas divididas. Los aislamientos realizados a partir de las muestras intestinales de $E$. faecium presentaron un mayor número de poblaciones celulares del sistema inmune a nivel intestinal $(p<0.01)$. Además, se realizó medición del pH intestinal, el cual presentó una disminución estadística significativa $(\mathrm{p}<0.01)$, donde los animales que consumieron $E$. faecium presentaron los valores más bajos de $\mathrm{pH}$ intestinal, frente a aquellos que consumieron la dieta con adición de antibiótico. La adición de la cepa $E$. faecium en el alimento de lechones recién destetados, estimula el aumento en el número de poblaciones celulares intestinales del sistema inmune y alcanzan una mayor disminución del pH intestinal.

\section{Addition of Enterococcus faecium increases intestinal immune response in growing pigs}

\section{SUMMARY}

\section{ADDITIONAL KEYWORDS}

Wean.

Piglets.

Probiotics.

Immune system.

\section{INFORMACIÓN}

Cronología del artículo.

Recibido/Received: 28.09.2015

Aceptado/Accepted: 22.06.2016

On-line: 15.09 .2016

Correspondencia a los autores/Contact e-mail:

vhherrer@unal.edu.co

\section{INTRODUCCIÓN}

El destete en los lechones es catalogado como un momento crítico de su crianza, y en el que se pone en riesgo la viabilidad del animal. Al momento del destete se presenta un estrés alimentario, debido a que los animales pasan de consumir mayoritariamente leche a consumir un alimento complejo; y un estrés social, causado por la separación abrupta de la madre y la reagrupación de lechones en transición (Gutiérrez et al., 2012; Montoya et al., 2012). Además, el cambio de dieta provoca un aumento del $\mathrm{pH}$ en el tracto gastrointestinal, debido a la disminución de la producción de ácido láctico a partir de lactosa de la leche, y al mayor contenido de proteína que actúa tamponando la escasa capacidad acidificante del inmaduro tracto digestivo del lechón. El elevado $\mathrm{pH}$ favorece la supervivencia y proliferación de la microbiota patógena, principalmente de los serotipos de E. coli que producen diferentes tipos de enterotoxinas (STa, STb y EAST1) (SuárezBelloch y Latorre, 2014), afectando negativamente a la pared intestinal y provocando la hipersecreción de 
Tabla I. Análisis proximal de la dieta basal (Proximate analysis of the basal diet)

\begin{tabular}{lc}
\hline Proteína bruda (\%) & 22,0 \\
Extracto etéreo (\%) & 6,0 \\
Cenizas (\%) & 8,0 \\
Fibra (\%) & 5,0 \\
Humedad (\%) & 13,0 \\
\hline
\end{tabular}

agua y electrolitos. Todo lo anterior conlleva a una pobre respuesta inmunitaria, específicamente en animales jóvenes en los que la inmunidad materna ha desaparecido (Suárez-Belloch y Latorre, 2014).

El intestino es un importante compartimiento del tracto gastrointestinal (TGI) donde se alberga una microbiota compleja y se desarrolla el sistema inmune de la mucosa (Heo et al., 2013). El epitelio intestinal está constantemente expuesto a altos niveles de antígenos alimenticios y bacterianos. El intestino y el tejido linfoide asociado al intestino (GALT, por sus siglas en inglés) son componentes esenciales de la defensa inmune, protegiendo al organismo de antígenos y patógenos extraños, mientras tolera bacterias comensales y antígenos de la dieta (Walker et al., 2012).

El sistema inmune de la mucosa intestinal realiza una doble labor: identificar nutrientes inocuos y eliminar alguna respuesta inmune sistémica que pudiera ejercerse contra ellos. De igual manera, este sistema reacciona con el fin de eliminar alguna invasión por bacterias, virus, parásitos y hongos (Boehm et al., 2012). El desbalance de este equilibrio puede contribuir a numerosas patogénesis de condiciones inflamatorias (Walker et al., 2012).

Dentro de los procedimientos utilizados para prevenir las infecciones entéricas durante la etapa del destete, se encuentra el suministro de antibióticos. A pesar de que la utilización de estos productos asegura el control de infecciones bacterianas (Jurado et al., 2013), la aplicación de dosis inadecuadas e indiscriminadas generan la aparición de cepas resistentes, cada vez más patógenas y con implicaciones negativas para la salud animal y humana, debido principalmente a los niveles residuales de dichos antibióticos en carne (Martin et al., 2012; Allen et al., 2012).

Como una alternativa al uso de antibióticos en contra de infecciones bacterianas se ha propuesto diferentes estrategias terapéuticas de origen nutricional, entre ellas la utilización de microorganismos vivos que mejoran el equilibrio microbiano intestinal y que aportan beneficios a la salud del animal. Estos microorganismos son denominados probióticos, dentro de los cuales las especies más representativas son Lactobacillus spp (Saad et al., 2013).

En el campo de la producción porcina los probióticos han sido útiles para tratar diferentes enfermedades entéricas. Algunos estudios ponen de manifiesto que éstos ayudan a prevenir y curar diarreas infecciosas (Deegan et al., 2006; Zacharof y Lovitt, 2012), debido a que favorecen el desarrollo y la estabilidad de la microflora, y la estimulación del sistema inmunológico (Hansen et al., 2012). Además, estos microorganismos proporcionan nutrientes a las células intestinales, promueven la absorción de nutrientes, y crean un ambiente intestinal beneficioso (Bauer et al., 2006). Por esto, el objetivo de este trabajo fue determinar el efecto de la adición de cepas de probióticos en cerdos en crecimiento sobre las poblaciones celulares del sistema inmune (eosinófilos, basófilos, neutrófilos, monocitos y linfocitos) a nivel intestinal.

\section{MATERIAL Y MÉTODOS}

\section{CONSIDERACIONES ÉTICAS}

Todos los procedimientos experimentales fueron llevados a cabo de acuerdo a las guías propuestas por The International Guiding Principles for Biomedical Research Involving Animals (CIOMS, 1985). Esta investigación fue avalada por El Comité de Ética en la Experimentación Animal de la Universidad Nacional de Colombia, Sede Medellín (CEMED- 012 del 14 de Mayo de 2012).

\section{LOCALIZACIÓN}

El trabajo de campo se realizó bajo condiciones comerciales en la Granja Caña Brava, ubicada en el municipio de Gómez Plata (Antioquia, Colombia), vereda La Bonita, localizada a 1540 metros sobre el nivel del mar (m.s.n.m), con una temperatura promedio de $21^{\circ} \mathrm{C}$, perteneciente a la zona de vida de Bosque Húmedo Premontano (bh-PM).

\section{ANIMALES}

Se sacrificaron 35 lechones (hembras y machos) del cruce duroc $x$ pietran, destetados exactamente a los 21 días de edad, con un peso aproximado de $6 \pm 0.5 \mathrm{Kg}$. Cada una de las instalaciones o corrales estaba provista de comederos de canoa y bebedero automático con agua a voluntad, y contaba con temperatura controlada $\left(26 \pm 3^{\circ} \mathrm{C}\right)$. La dieta comercial ofrecida fue enriquecida con vitaminas, minerales y lisina HCL. Las dietas se balancearon para cumplir con todos los mínimos nutricionales requeridos y propuestos por el NRC (2012) (tabla I). La cantidad de alimento ofrecido a los lechones por jaula fue administrada de acuerdo a la tabla dietaria que corresponde para la etapa productiva (levante). Así mismo, el agua de bebida que contenía las cepas probióticas se ofreció diariamente desde el día 1 del destete hasta finalizar el experimento, el cual tuvo una duración de 30 días (correspondiente a la fase de levante). Durante la lactancia no se suministró alimento sólido a los lechones.

\section{INSTALACIONES Y EQUIPOS}

Las instalaciones fueron desinfectadas y encaladas para la llegada de los lechones. Los cerdos fueron alojados en corrales con suelo de cemento (1.5 x 3 metros) y desde el día 0 al 15 del experimento, los corrales estaban dotados de lechoneras y cama de viruta de madera. Para mantener la temperatura homogénea el corral estaba provisto de cortinas. 
Para realizar el pesaje de los cerdos y el alimento suministrado se utilizó una balanza digital.

\section{DiETAS}

Los animales fueron aleatorizados a una de dos dietas: dieta comercial con y sin la adición de antibiótico. Los diferentes probióticos (Lactobacillus casei ATCC393, Lactobacillus acidophilus ATCC4356 o Enterococcus faecium ATCC35667) se suministraron en el agua de bebida de los animales que consumieron la dieta comercial sin antibiótico, así:

Dieta 1 Control (D1): Alimento comercial sin antibiótico, sin adición de cepa probiótica en el agua de bebida.

Dieta 2 (D2): Alimento comercial con antibiótico, sin adición de cepa probiótica en el agua de bebida.

Dieta 3 (D3): Alimento comercial sin antibiótico, con adición de la cepa comercial probiótica L. acidophilus en el agua de bebida.

Dieta 4 (D4): Alimento comercial sin antibiótico, con adición de la cepa comercial probiótica L. casei en el agua de bebida.

Dieta 5 (D5): Alimento comercial sin antibiótico, con adición de la cepa comercial probiótica E. faecium en el agua de bebida.

La cantidad del probiótico adicionado (relación $\mathrm{v} / \mathrm{p}$ ) fue del $20 \%$ con respecto al peso de la ración utilizada, siguiendo las instrucciones para su preparación y adición según lo recomendado por el fabricante. La inclusión de los probióticos en el agua de bebida se realizó (dos veces al día) por mezclado directo de un litro de agua con 30 gramos de azúcar comercial, la cual fue adicionada en un tanque de 20 lt de agua, y evaluada por medio de análisis microbiológicos semanales para garantizar poblaciones mínimas de $10^{8}$ $\mathrm{UFC} / \mathrm{ml}$ con una viabilidad adecuada. El alimento utilizado en el estudio estuvo libre de antibióticos (excepto la dieta D2), ya que no fue de interés modificar la dieta, sino la incorporación de los probióticos como una alternativa al uso de antibióticos. Las dietas experimentales se proporcionaron durante 30 días a partir del día del destete (día 21 de vida).

\section{SACRIFICIOS}

Durante la fase experimental se realizó eutanasia humanitaria a 35 lechones de la siguiente forma: el día inicial, o día 1 (día del destete), se sacrificaron 5 lechones, que representaron: el grupo de referencia para verificar el estado general de salud y la evaluación macroscópica del estado de los órganos de los animales antes de suministrar las dietas experimentales, y las unidades experimentales para cada uno de los tratamientos. Los días 15 y 30 posdestete fueron sacrificados 3 lechones al azar de cada tratamiento. Todos los lechones se sacrificaron 2.5 horas después de su última comida. Los animales se sedaron con un neuroléptico (stresnil@ (Azaperona)) vía intramuscular, y posteriormente se sometieron a inhalación con Nitrox®.

Tabla II. Poblaciones de células inmunes en vellosidades intestinales de cerdos, con y sin adición de cepas probióticas (Immune cell populations in intestinal villi of pigs with and without addition of probiotic strains).

\begin{tabular}{|c|c|c|c|c|c|c|c|c|}
\hline Ubicación & $\begin{array}{l}\text { Poblaciones } \\
\text { celulares }\end{array}$ & Días & D1 & D2 & D3 & D4 & D5 & EEM \\
\hline \multirow{18}{*}{$\begin{array}{l}0 \\
\frac{0}{0} \\
\frac{\pi}{0} \\
\frac{0}{0} \\
\frac{0}{0} \\
\end{array}$} & \multirow[t]{3}{*}{ Neutrófilos } & 1 & $0,039^{x}$ & $0,038^{x}$ & $0,038^{x}$ & $0,037^{x}$ & $0,038^{x}$ & \multirow{18}{*}{0,003} \\
\hline & & 15 & $0,051^{A, Y}$ & $0,064^{B, Y}$ & $0,065^{B, Y}$ & $0,083^{C, Y}$ & $0,099^{c, Y}$ & \\
\hline & & 30 & $0,064^{A, Z}$ & $0,076^{\mathrm{B}, \mathrm{Z}}$ & $0,085^{c, z}$ & $0,099^{\mathrm{D}, \mathrm{z}}$ & $0,114^{\mathrm{E}, Z}$ & \\
\hline & \multirow[t]{3}{*}{ Eosinófilos } & 1 & $0,43^{x}$ & $0,42^{x}$ & $0,43^{x}$ & $0,42^{x}$ & $0,431^{x}$ & \\
\hline & & 15 & $0,45^{\mathrm{A}, \mathrm{Y}}$ & $0,48^{B, Y}$ & $0,51^{\mathrm{C}, \mathrm{Y}}$ & $0,57^{\mathrm{D}, \mathrm{Y}}$ & $0,59^{E, Y}$ & \\
\hline & & 30 & $0,49^{A, Z}$ & $0,54^{\mathrm{B}, \mathrm{Z}}$ & $0,57^{c, z}$ & $0,63^{\mathrm{D}, \mathrm{Z}}$ & $0,70^{\mathrm{E}, Z}$ & \\
\hline & \multirow[t]{3}{*}{ Macrófagos } & 1 & $0,41^{x}$ & $0,40^{x}$ & $0,39^{x}$ & $0,40^{x}$ & $0,41^{x}$ & \\
\hline & & 15 & $0,43^{A, Y}$ & $0,45^{\mathrm{B}, \mathrm{Y}}$ & $0,48^{\mathrm{C}, \mathrm{Y}}$ & $0,52^{\mathrm{D}, \mathrm{Y}}$ & $0,55^{\mathrm{E}, \mathrm{Y}}$ & \\
\hline & & 30 & $0,51^{A, Z}$ & $0,54^{\mathrm{B}, \mathrm{Z}}$ & $0,57^{c, z}$ & $0,61^{\mathrm{D}, \mathrm{Z}}$ & $0,66^{\mathrm{E}, \mathrm{Z}}$ & \\
\hline & \multirow[t]{3}{*}{ Linfocitos } & 1 & $3,01^{x}$ & $3,02^{x}$ & $3,03^{x}$ & $3,02^{x}$ & $3,01^{x}$ & \\
\hline & & 15 & $3,95^{\mathrm{A}, \mathrm{Y}}$ & $4,04^{\mathrm{B}, \mathrm{Y}}$ & $4,24^{\mathrm{C}, \mathrm{Y}}$ & $4,54^{\mathrm{D}, \mathrm{Y}}$ & $4,95^{\mathrm{E}, \mathrm{Y}}$ & \\
\hline & & 30 & $4,18^{A, Z}$ & $4,48^{\mathrm{B}, \mathrm{Z}}$ & $4,79^{c, z}$ & $4,99^{\mathrm{D}, \mathrm{z}}$ & $5,21^{\mathrm{E}, Z}$ & \\
\hline & \multirow[t]{3}{*}{ Plasmocitos } & 1 & $0,186^{x}$ & $0,188^{x}$ & $0,184^{x}$ & $0,185^{x}$ & $0,187^{x}$ & \\
\hline & & 15 & $0,204^{A, Y}$ & $0,224^{B, Y}$ & $0,245^{\mathrm{C}, Y}$ & $0,268^{D, Y}$ & $0,294^{E, Y}$ & \\
\hline & & 30 & $0,228^{A, Z}$ & $0,241^{\mathrm{B}, \mathrm{Z}}$ & $0,263^{c, z}$ & $0,294^{\mathrm{D}, \mathrm{z}}$ & $0,318^{\mathrm{E}, \mathrm{Z}}$ & \\
\hline & \multirow{3}{*}{$\begin{array}{l}\text { Linfocitos } \\
\text { Granulares }\end{array}$} & 1 & $0,044^{x}$ & $0,041^{x}$ & $0,043^{x}$ & $0,047^{x}$ & $0,045^{x}$ & \\
\hline & & 15 & $0,056^{A, Y}$ & $0,069^{B, Y}$ & $0,084^{\mathrm{C}, \mathrm{Y}}$ & $0,099^{D, Y}$ & $0,112^{E, Y}$ & \\
\hline & & 30 & $0,074^{A, Z}$ & $0,088^{\mathrm{B}, \mathrm{Z}}$ & $0,102^{c, z}$ & $0,125^{\mathrm{D}, \mathrm{Z}}$ & $0,152^{D, Y}$ & \\
\hline
\end{tabular}

D1: Alimento comercial sin antibiótico y sin probiótico; D2: Alimento comercial + antibiótico; D3: Alimento comercial sin antibiótico + L. acidophilus; D4: Alimento comercial sin antibiótico + L. casei; D5: Alimento comercial sin antibiótico + E. faecium. A,B,C,D,E Dentro de una misma fila, medias con diferente superíndice son estadísticamente diferentes $(p<0,05)$. ${ }^{x, Y}, \mathrm{Dentro}$ de una misma columna, medias con un superíndice común (por variable en estudio) no difieren estadísticamente $(p<0,05)$. EEM: Error estándar de la media. 
Tabla III. Poblaciones de células inmunes en criptas intestinales de cerdos, con y sin adición de cepas probióticas (Immune cell populations in intestinal crypts of pigs with and without addition of probiotic strains).

\begin{tabular}{|c|c|c|c|c|c|c|c|c|}
\hline Ubicación & Poblaciones celulares & Días & D1 & D2 & D3 & D4 & D5 & EEM \\
\hline \multirow{18}{*}{ 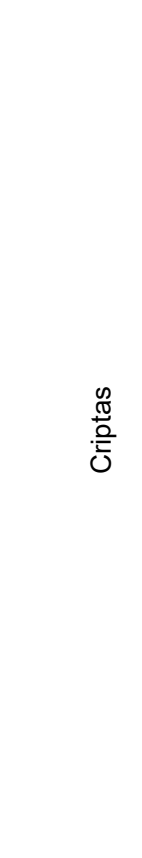 } & Neutrófilos & 1 & $0,037^{x}$ & $0,039^{x}$ & $0,041^{x}$ & $0,038^{x}$ & $0,040^{x}$ & \multirow{18}{*}{0,003} \\
\hline & & 15 & $0,048^{A, Y}$ & $0,054^{A, Y}$ & $0,067^{\mathrm{B}, Y}$ & $0,081^{C, Y}$ & $0,099^{D, Y}$ & \\
\hline & & 30 & $0,059^{A, Z}$ & $0,076^{\mathrm{B}, \mathrm{Z}}$ & $0,089^{c, z}$ & $0,102^{\mathrm{D}, \mathrm{Z}}$ & $0,117^{\mathrm{D}, \mathrm{Z}}$ & \\
\hline & Eosinófilos & 1 & $0,171^{x}$ & $0,176^{x}$ & $0,175^{x}$ & $0,172^{x}$ & $0,175^{x}$ & \\
\hline & & 15 & $0,192^{A, Y}$ & $0,223^{B, Y}$ & $0,254^{\mathrm{C}, \mathrm{Y}}$ & $0,277^{\mathrm{D}, \mathrm{Y}}$ & $0,299^{E, Y}$ & \\
\hline & & 30 & $0,241^{A, Z}$ & $0,259^{\mathrm{B}, \mathrm{Z}}$ & $0,283^{c, z}$ & $0,317^{D, Z}$ & $0,341^{E, Z}$ & \\
\hline & Macrófagos & 1 & $0,351^{x}$ & $0,360^{x}$ & $0,354^{x}$ & $0,355^{x}$ & $0,351^{x}$ & \\
\hline & & 15 & $0,390^{A, Y}$ & $0,431^{\mathrm{B}, \mathrm{Y}}$ & $0,475^{\mathrm{C}, Y}$ & $0,510^{\mathrm{D}, Y}$ & $0,558^{\mathrm{E}, \mathrm{Y}}$ & \\
\hline & & 30 & $0,521^{A, Z}$ & $0,597^{\mathrm{B}, \mathrm{Z}}$ & $0,623^{c, z}$ & $0,649^{D, z}$ & $0,695^{\mathrm{E}, \mathrm{Z}}$ & \\
\hline & Linfocitos & 1 & $2,31^{x}$ & $2,30^{x}$ & $2,31^{x}$ & $2,30^{x}$ & $2,311^{x}$ & \\
\hline & & 15 & $2,56^{\mathrm{A}, Y}$ & $2,94^{\mathrm{B}, \mathrm{Y}}$ & $3,19^{\mathrm{C}, \mathrm{Y}}$ & $3,35^{\mathrm{D}, \mathrm{Y}}$ & $3,52^{\mathrm{E}, \mathrm{Y}}$ & \\
\hline & & 30 & $2,94^{A, Z}$ & $3,09^{\mathrm{B}, \mathrm{z}}$ & $3,35^{c, z}$ & $3,56^{\mathrm{D}, \mathrm{Z}}$ & $3,74^{\mathrm{E}, \mathrm{Z}}$ & \\
\hline & Plasmocitos & 1 & $0,037^{x}$ & $0,041^{x}$ & $0,039^{x}$ & $0,042^{x}$ & $0,037^{x}$ & \\
\hline & & 15 & $0,054^{A, Y}$ & $0,068^{B, Y}$ & $0,087^{\mathrm{C}, \mathrm{Y}}$ & $0,105^{\mathrm{D}, Y}$ & $0,123^{E, Y}$ & \\
\hline & & 30 & $0,079^{A, Z}$ & $0,098^{\mathrm{B}, \mathrm{Z}}$ & $0,117^{c, z}$ & $0,124^{\mathrm{D}, \mathrm{Z}}$ & $0,141^{\mathrm{E}, \mathrm{Z}}$ & \\
\hline & Linfocitos Granulares & 1 & $0,41^{x}$ & $0,40^{x}$ & $0,41^{x}$ & $0,40^{x}$ & $0,41^{x}$ & \\
\hline & & 15 & $0,48^{A, Y}$ & $0,58^{\mathrm{B}, \mathrm{Y}}$ & $0,68^{\mathrm{C}, \mathrm{Y}}$ & $0,72^{\mathrm{D}, \mathrm{Y}}$ & $0,75^{\mathrm{E}, \mathrm{Y}}$ & \\
\hline & & 30 & $0,52^{A, Z}$ & $0,63^{\mathrm{B}, \mathrm{Z}}$ & $0,74^{\mathrm{c}, \mathrm{z}}$ & $0,81^{\mathrm{D}, \mathrm{Z}}$ & $0,84^{\mathrm{E}, Z}$ & \\
\hline
\end{tabular}

D1: Alimento comercial sin antibiótico y sin probiótico; D2: Alimento comercial + antibiótico; D3: Alimento comercial sin antibiótico + L. acidophilus; D4: Alimento comercial sin antibiótico + L. casei; D5: Alimento comercial sin antibiótico + E. faecium. A,B,C,D,E Dentro de una misma fila, medias con diferente superíndice son estadísticamente diferentes $(p<0,05)$. $x, y, Z$ Dentro de una misma columna, medias con un superíndice común (por variable en estudio) no difieren estadísticamente $(p<0,05)$. EEM: Error estándar de la media.

Después del sacrificio, los lechones se colocaron en posición decúbito dorsal, se diseccionó la región abdominal y se extrajo completamente el intestino delgado desde la unión pilórica hasta la válvula íleo-cecal (Segalés y Domingo, 2003). El intestino fue alineado y medido en una mesa sin ningún tipo de tensión. Posteriormente éste se dividió en tres regiones (duodeno, yeyuno, e íleon), y se tomaron $20 \mathrm{~cm}$ de cada sección. Una vez cortadas las porciones, la digesta contenida en cada una de ellas se removió mediante lavado por infusión con solución salina fría según lo descrito previamente (Reis et al., 2005). Seguidamente, estas porciones fueron incluidas en formalina tamponada al $10 \%$ hasta realizar las determinaciones de morfometría e histopatología.

\section{TOMA DE MUESTRA Y DETERMINACIÓN DEL PH INTESTINAL}

Se tomó $1 \mathrm{~g}$ de muestra (mezcla) de contenido de duodeno, yeyuno e íleon, para luego suspenderlo en $12,5 \mathrm{ml}$ de agua destilada desionizada. Esta mezcla se agitó manualmente con agitador de vidrio lavándolo en cada registro con agua destilada. Posteriormente se insertó en la mezcla un electrodo de $\mathrm{pH}$ y se realizaron las lecturas en un potenciómetro con precisión de tres decimales. El pH de la suspensión fue medido dentro de los 45 minutos subsiguientes al sacrificio de los lechones (Jaramillo, 2011).

\section{ANÁLISIS DE MUESTRAS}

Después de 24 horas de la colecta, las muestras obtenidas de duodeno, yeyuno e íleon fueron enviadas al laboratorio para ser analizadas por histopatólogos expertos.

Los tejidos fueron fijados en formalina tamponada al $10 \%$ durante 24 horas a $4^{\circ} \mathrm{C}$, incluidos en parafina, cortados a $4 \mu \mathrm{m}$ de espesor y coloreados con hematoxilina-eosina para ser lavados y almacenados en etanol: agua $(75: 25, \mathrm{v}: \mathrm{v})$ de acuerdo con el método descrito por Nabuurs et al. (1993). El análisis de imágenes histológicas se realizó ciñendo la observación a los siguientes parámetros:

\section{Área a analizar: $63000 \mu \mathrm{m}^{2}$}

\section{Número de áreas / fotografía: 2}

El conteo celular: leucocitos (unidad de área), neutrófilos, eosinófilos, macrófagos, linfocitos, plasmocitos y linfocitos granulares

\section{ANÁLISIS ESTADÍSTICO}

Se realizó según un diseño de bloques al azar en un arreglo de parcelas divididas. Para la conformación de los bloques se tomó en consideración el peso inicial de los animales. A cada animal le fue asignado uno de los 15 tratamientos ( 5 dietas experimentales y tres periodos de evaluación). Cada tratamiento tuvo un total de 3 repeticiones. El análisis estadístico se realizó según el procedimiento GLM (modelos lineales generales) del SAS (2007). Las diferencias entre las medias de los tratamientos fueron determinadas por mínimos cuadrados y analizadas por ANOVA. Para realizar la comparación de los promedios entre tratamientos se utilizó una prueba de Duncan $(\mathrm{p}<0.05)$. 
Tabla IV. Poblaciones de células inmunes en vellosidades y criptas intestinales de cerdos, con y sin adición de cepas probióticas, según el segmento intestinal (Immune cell populations in intestinal villi and crypts of pigs with and without addition of probiotic strains according to the intestinal segment).

\begin{tabular}{|c|c|c|c|c|c|}
\hline \multirow{2}{*}{$\begin{array}{l}\text { Variables } \\
\text { Duodeno }\end{array}$} & & \multicolumn{3}{|c|}{ Segmento intestinal } & \multirow[t]{2}{*}{ EEM } \\
\hline & & Duodeno & Yeyuno & Íleon & \\
\hline \multirow[t]{2}{*}{ Neutrófilos } & Vellosidades & $0,071^{\mathrm{A}}$ & $0,074^{\mathrm{A}}$ & $0,091^{\text {в }}$ & 0,003 \\
\hline & Criptas & $0,080^{A}$ & $0,084^{\mathrm{A}}$ & $0,098^{B}$ & \\
\hline \multirow[t]{2}{*}{ Eosinófilos } & Vellosidades & $0,526^{A}$ & $0,545^{\mathrm{A}}$ & $0,637^{\mathrm{B}}$ & 0,008 \\
\hline & Criptas & $0,271^{A}$ & $0,274^{\mathrm{A}}$ & $0,318^{\mathrm{B}}$ & \\
\hline \multirow[t]{2}{*}{ Macrófagos } & Vellosidades & $0,514^{\mathrm{A}}$ & $0,529^{A}$ & $0,579^{\mathrm{B}}$ & 0,009 \\
\hline & Criptas & $0,604^{A}$ & $0,613^{A}$ & $0,648^{\mathrm{B}}$ & \\
\hline \multirow[t]{2}{*}{ Linfocitos } & Vellosidades & $3,97^{A}$ & $4,03^{\mathrm{A}}$ & $5.43^{B}$ & 0,050 \\
\hline & Criptas & $3,22^{A}$ & $3,25^{A}$ & $3.67^{\mathrm{B}}$ & \\
\hline \multirow[t]{2}{*}{ Plasmocitos } & Vellosidades & $0,240^{A}$ & $0,244^{\mathrm{B}}$ & $0,288^{\mathrm{B}}$ & 0,004 \\
\hline & Criptas & $0,105^{\mathrm{A}}$ & $0,110^{\mathrm{A}}$ & $0,125^{\mathrm{B}}$ & \\
\hline \multirow[t]{2}{*}{ Linfocitos Granulares } & Vellosidades & $0,089^{A}$ & $0,096^{A}$ & $0,117^{\mathrm{B}}$ & 0,003 \\
\hline & Criptas & $0,693^{A}$ & $0,703^{\mathrm{A}}$ & $0,783^{\mathrm{B}}$ & \\
\hline
\end{tabular}

D1: Alimento comercial sin antibiótico y sin probiótico; D2: Alimento comercial + antibiótico; D3: Alimento comercial sin antibiótico + L. acidophilus; D4: Alimento comercial sin antibiótico + L. casei; D5: Alimento comercial sin antibiótico + E. faecium. A,B,C,D,E Dentro de una misma fila, medias con diferente superíndice son estadísticamente diferentes $(p<0,05)$. ${ }^{x, y, Z}$ Dentro de una misma columna, segmentos intestinales con un superíndice común no difieren estadísticamente $(p>0,05)$. EEM: Error estándar de la media.

\section{RESULTADOS}

Los cerdos que consumieron las dietas experimentales no presentaron signo alguno de enfermedad que causara su retiro o sacrificio inmediato. Además, al nivel en que se fijó el suministro diario de alimento y agua no generó sobrantes.

En este experimento no se encontró interacción estadística entre las diferentes dietas experimentales dentro de cada período de evaluación para ninguna de las variables en estudio, por lo que no fue necesario analizar y desglosar dichos factores de manera independiente.
Los cambios de las poblaciones celulares del sistema inmune estudiadas en las vellosidades intestinales entre cada una de las dietas y periodos de exposición se pueden observar en la tabla II. Las poblaciones celulares presentaron un aumento estadístico significativo $(p<0,01)$ entre las diferentes dietas evaluadas dentro de cada período de evaluación, donde D1 obtuvo valores menores respecto a D2 y frente a los tratamientos con probióticos, donde los animales del tratamiento D5 mostraron los valores más altos en las poblaciones de células del sistema inmune intestinal. Para las mismas variables en estudio, se presentó diferencia significativa estadística entre los diferentes días de muestreo dentro de cada una de las dietas $(\mathrm{p}<0,05)$, donde en el día 30

Tabla V. pH intestinal en cerdos, con y sin adición de cepas probióticas (Intestinal pH in pigs, with and without addition of probiotic strains)

\begin{tabular}{|c|c|c|c|c|c|c|c|}
\hline $\begin{array}{l}\text { Segmento } \\
\text { intestinal }\end{array}$ & Día & D1 & $\mathrm{D} 2$ & D3 & D4 & D5 & EEM \\
\hline \multirow[t]{3}{*}{ Duodeno 1} & 1 & $6,78^{x}$ & $6,79^{x}$ & $6,76^{x}$ & $6,78^{x}$ & $6,77^{x}$ & \\
\hline & 15 & $6,63^{A, Y}$ & $6,52^{\mathrm{B}, Y}$ & $6,45^{\mathrm{C}, Y}$ & $6,34^{\mathrm{D}, \mathrm{Y}}$ & $6,29^{E, Y}$ & \\
\hline & 30 & $6,54^{\mathrm{A}, \mathrm{Z}}$ & $6,45^{\mathrm{B}, \mathrm{Z}}$ & $6,37^{c, z}$ & $6,31^{\mathrm{D}, \mathrm{Z}}$ & $6,25^{\mathrm{E}, Z}$ & \\
\hline \multirow[t]{3}{*}{ Yeyuno $^{2}$} & 1 & $6,55^{x}$ & $6,54^{x}$ & $6,55^{x}$ & $6,53^{x}$ & $6,54^{x}$ & \\
\hline & 15 & $6,39^{A, Y}$ & $6,29^{B, Y}$ & $6,24^{\mathrm{C}, Y}$ & $6,25^{\mathrm{D}, Y}$ & $6,17^{E, Y}$ & 0,005 \\
\hline & 30 & $6,23^{A, Z}$ & $6,18^{\mathrm{B}, \mathrm{Z}}$ & $6,15^{\mathrm{c}, \mathrm{Z}}$ & $6,09^{D, Z}$ & $6,03^{E, Z}$ & \\
\hline \multirow[t]{3}{*}{ Íleon ${ }^{3}$} & 1 & $6,45^{x}$ & $6,44^{x}$ & $6,44^{x}$ & $6,45^{x}$ & $6,44^{x}$ & \\
\hline & 15 & $6,27^{A, Y}$ & $6,21^{\mathrm{B}, Y}$ & $6,14^{\mathrm{C}, \mathrm{Y}}$ & $6,08^{\mathrm{D}, Y}$ & $6,01^{\mathrm{E}, Y}$ & \\
\hline & 30 & $6,19^{A, Z}$ & $6,12^{\mathrm{B}, \mathrm{Z}}$ & $6,07^{c, z}$ & $6,01^{\mathrm{D}, \mathrm{Z}}$ & $5,87^{E, Z}$ & \\
\hline
\end{tabular}

D1: Alimento comercial sin antibiótico y sin probiótico; D2: Alimento comercial + antibiótico; D3: Alimento comercial sin antibiótico + L. acidophilus; D4: Alimento comercial sin antibiótico + L. casei; D5: Alimento comercial sin antibiótico + E. faecium. A,B,C,D,E Dentro de una misma fila, medias con diferente superíndice son estadísticamente diferentes $(p<0,05)$. $x, y, z$ Dentro de una misma columna, medias con un superíndice común (por variable en estudio) no difieren estadísticamente ( $p<0.05)$. $^{1,2,3}$ Dentro de una misma columna con un superíndice común (por variable en estudio) no difieren estadísticamente $(p<0,05)$. EEM: Error estándar de la media. 
se presentaron los mayores valores para cada dieta en estudio.

Las figuras 1 a 6 , ilustran el comportamiento de las poblaciones de células del sistema inmune en las vellosidades intestinales, de acuerdo a las diferentes dietas experimentales durante el tiempo del experimento.

En relación al parámetro de las poblaciones celulares inmunes estudiadas en las criptas intestinales entre cada una de las dietas y periodos de exposición, los cambios se pueden observar en la tabla III. Las poblaciones celulares presentaron un aumento estadístico significativo $(p<0,01)$ entre las diferentes dietas evaluadas dentro de cada período de evaluación, donde D1 obtuvo valores menores respecto a D2 y frente a las dietas con probióticos, donde los animales en D5 mostraron los valores más altos en las poblaciones de células intestinales. Para las mismas variables en estudio se presentó diferencia significativa estadística entre los diferentes días de muestreo dentro de cada una de las dietas $(\mathrm{p}<0,05)$, donde en el día 30 se presentaron los mayores valores para cada dieta en estudio.

Las figuras 7 a 12, ilustran el comportamiento de las poblaciones celulares en las criptas intestinales, de acuerdo a las diferentes dietas durante el tiempo del experimento.

Para los segmentos intestinales se ha observado diferencia estadística significativa $(\mathrm{p}<0,01)$ para las poblaciones celulares intestinales, donde duodeno exhibió los valores más bajos en comparación con los otros dos segmentos (yeyuno e íleon) tanto en vellosidades como en criptas intestinales (tabla IV).

Las figuras 13 y 14, ilustran el comportamiento de células de acuerdo a los diferentes segmentos del TGI de los lechones, tanto en vellosidades (13) como en criptas intestinales (14), durante el tiempo del experimento.

Los cambios del $\mathrm{pH}$ intestinal determinado entre cada una de las dietas y periodos de exposición se pueden observar en la tabla $\mathbf{V}$. Con respecto al $\mathrm{pH}$ intestinal, se presentó una disminución estadística significativa $(\mathrm{p}<0,01)$ entre las diferentes dietas evaluadas, donde D1 obtuvo valores mayores respecto a D2 y frente a las dietas con probióticos, donde los animales en D5 reportaron los valores más bajos en el pH intestinal.

Las figuras 15 a 17, ilustran el comportamiento del $\mathrm{pH}$ intestinal de acuerdo a los dietas en los diferentes segmentos del TGI de los lechones, durante el período experimental.

\section{DISCUSIÓN}

La superficie de la mucosa de los mamíferos está en contacto directo con el medio ambiente por lo que están expuestos a antígenos. Las secreciones intestinales (enzimas, bacteriocinas, e inmunoglobulinas) en estas superficies están involucradas en la defensa del huésped junto a la secreción de anticuerpos de la mucosa (Abraham y Medzhitov, 2011). El epitelio intestinal está constantemente expuesto a grandes cantidades de antígenos alimenticios y bacterianos. En condiciones fisiológicas normales, la monocapa epitelial intestinal facilita un flujo controlado y selectivo de componentes entre el lúmen y la mucosa (Ghadimi et al., 2010). El balance entre la tolerancia y la inmunidad en el intestino está, en parte, determinada por poblaciones de células presentadoras de antígeno (CPA) en el intestino. El desbalance de este equilibrio puede contribuir a numerosas patogénesis de condiciones inflamatorias (Walker et al., 2012).

Existen varias células encargadas de la presentación de antígenos en el GALT, incluyendo los enterocitos y otras células epiteliales intestinales (CEI), tales como células $\mathrm{M}$, células dendríticas (CD), macrófagos y linfocitos T y B (Gómez-Llorente et al., 2010). Las bacterias activan las $C D$ directamente a través de los receptores de reconocimiento de patrones (PRR) o indirectamente por la captura de los productos apoptóticos/necróticos de otras células que están muriendo en respuesta a la exposición microbiana (Bermúdez-Brito et al., 2012). Como se observó en los resultados de la investigación actual, los lechones pertenecientes a los grupos suplementados con cepas probióticas, aumentaron el número de sus poblaciones intestinales debido en parte al destete y, además, a la inmunomodulación causada por la ingesta de las cepas probióticas. Según lo explicado por Galdeano (2007), la inmunomodulación ocurre simultáneamente de dos maneras: i) por la presencia de ingredientes de la nueva dieta y ii) por el consumo de bacterias probióticas que ayudan al equilibrio bacteriano y a la activación controlada del sistema inmune

Significativamente, en los días de muestreo de esta investigación, las poblaciones inmunes aumentaron, explicado esto por la acción ejercida por los probióticos y la preparación del sistema inmune del hospedador. Galdeano et al. (2013) demostraron que las bacterias probióticas indujeron un aumento en la celularidad de la lámina propia del intestino, principalmente en las células mononucleares, sin alterar la histología normal del intestino, donde los neutrófilos son las células predominantes.

La alteración de la histología normal del intestino, podría estar asociada a las respuestas inflamatorias e inmunes provocadas por diferentes manifestaciones de estrés durante el destete, dentro de las que se encuentran la separación abrupta de la madre, la reubicación en nuevos grupos sociales y el cambio a un alimento sólido (Mejía et al., 2012). Además, las respuestas fisiológicas al estrés del destete implican interacciones y respuestas complejas por parte de los sistemas nervioso central e inmunológico (Ospina et al., 2011); tal como se mostró en nuestra investigación, donde los lechones exhibieron alteraciones inmunológicas a nivel intestinal, evidenciando un aumento significativo de las poblaciones celulares inmunes intestinales a lo largo del experimento.

La manera en que se produce la activación del sistema inmune del intestino de manera controlada fue explicado por Galdeano et al. (2013), que demostraron que todas las bacterias probióticas interactúan con las células epiteliales intestinales (CEI), y los fragmentos bacterianos se internalizan por diferentes rutas de entrada (transcitosis) y hacen contacto con las células del sistema inmune, y a su vez, son capturados por la prolongación de células dendríticas en el lúmen o por 

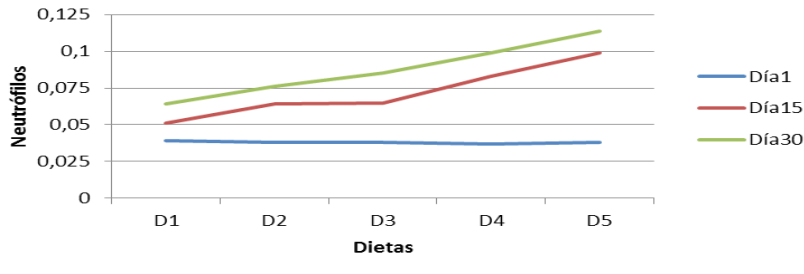

Figura 1. Neutrófilos en vellosidades de cerdos con y sin adición de cepa probiótica (Neutrophils in villi of pigs with and without adittion of probiotic strains).

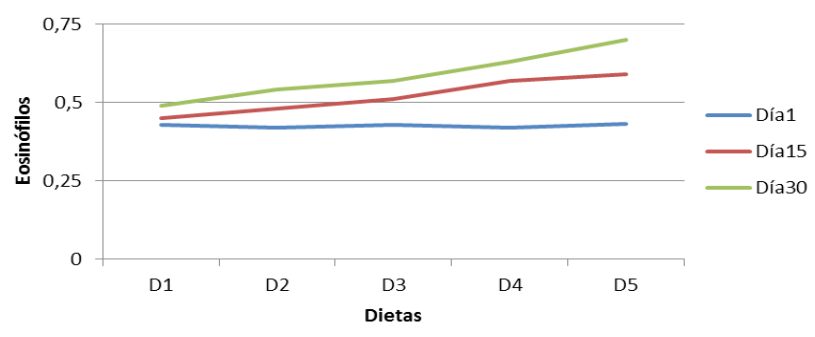

Figura 2. Eosinófilos en vellosidades de cerdos, con y sin adición de cepa probiótica (Eosinophils in villi of pigs with and without addition of probiotic strain).

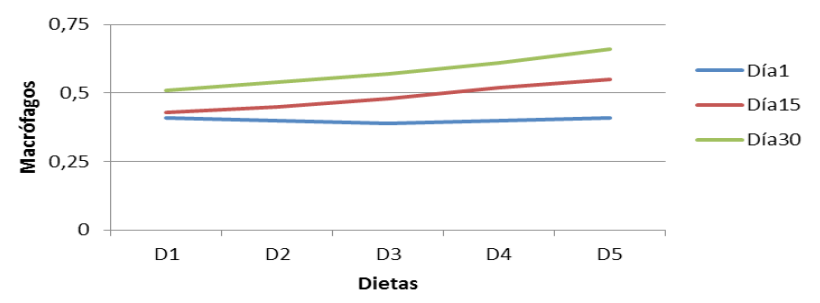

Figura 3. Macrófagos en vellosidades de cerdos, con y sin adición de cepa probiótica (Macrophages in villi of pigs with and without addition of probiotic strain).

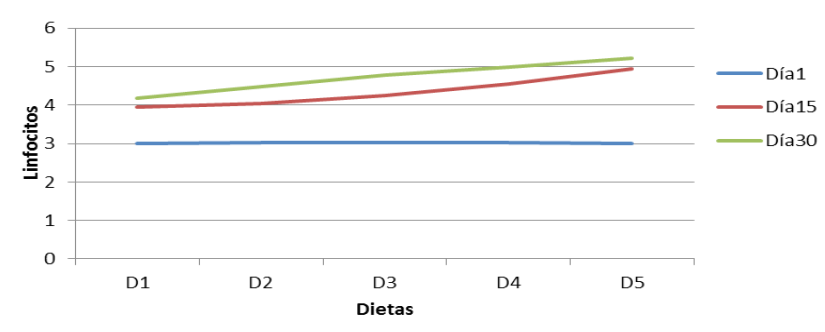

Figura 4. Linfocitos en vellosidades de cerdos, con y sin adición de cepa probiótica (Lymphocytes in villi of pigs with and without addition of probiotic strain).

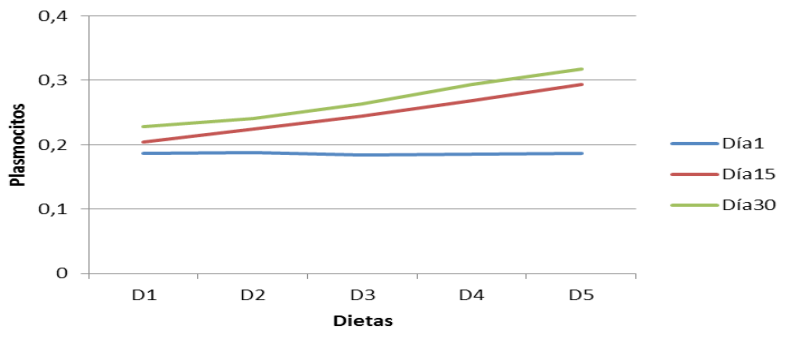

Figura 5. Plasmocitos en vellosidades de cerdos con y sin adición de cepa probiótica (Plasmocytes in villi of pigs with and without adittion of probiotic strains).

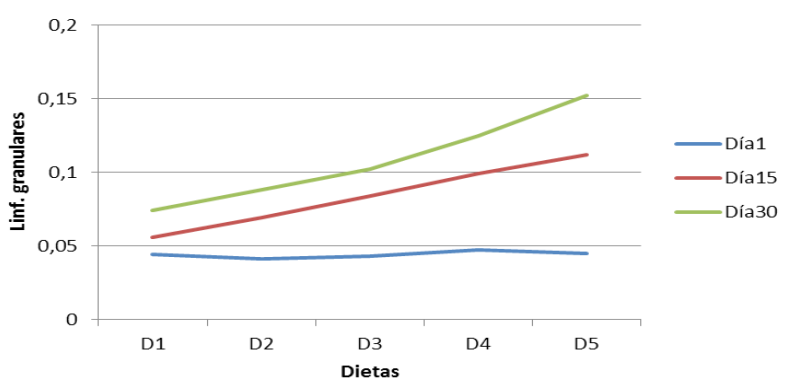

Figura 6. Linf. granulares Vellosidades de cerdos, con y sin adición de cepa probiótica (Granular lymphocytes in villi of pigs with and without addition of probiotic strain).

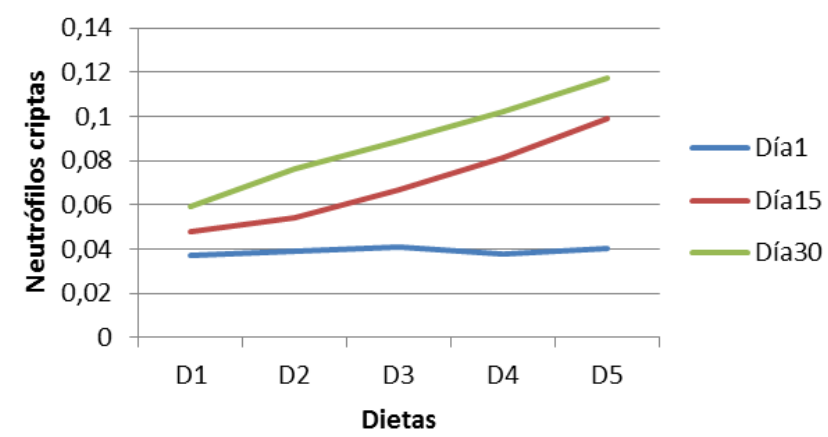

Figura 7. Neutrófilos en criptas de cerdos, con y sin adición de cepas probióticas (Neutrophil in crypts pigs with and without addition of probiotic strains).

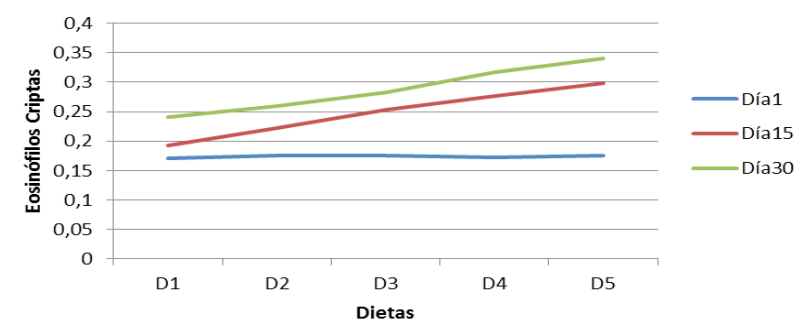

Figura 8. Eosinófilos en criptas de cerdos, con y sin adición de cepas probióticas (Eosinophils in crypts of pigs with and without addition of probiotic strains). 


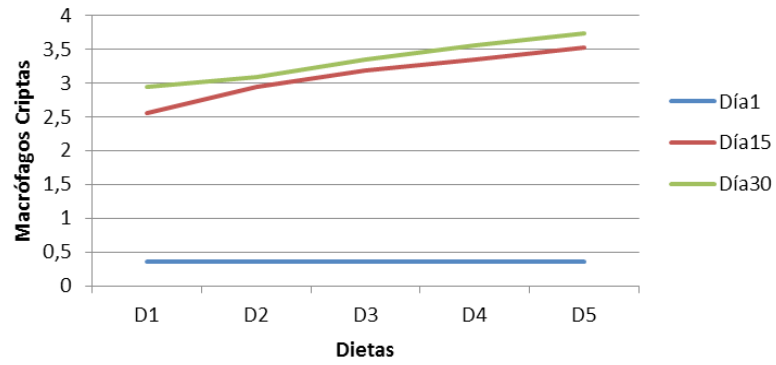

Figura 9. Macrófagos en criptas de cerdos, con y sin adición de cepas probióticas (Macrophages in crypts of pigs with and without addition of probiotic strains).

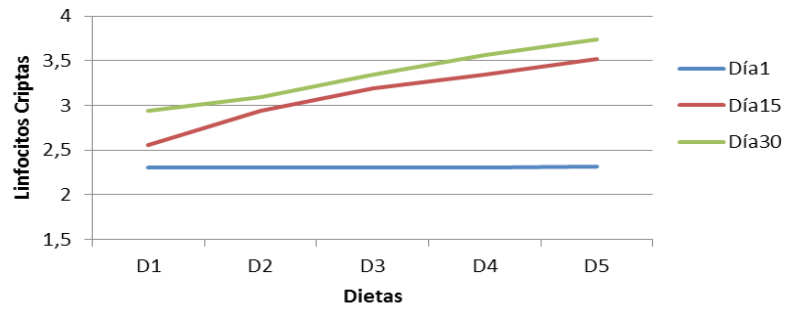

Figura 10. Linfocitos en criptas de cerdos con y sin adición de cepas probióticas (Lymphocytes in crypts of pigs with and without addition of probiotic strains).

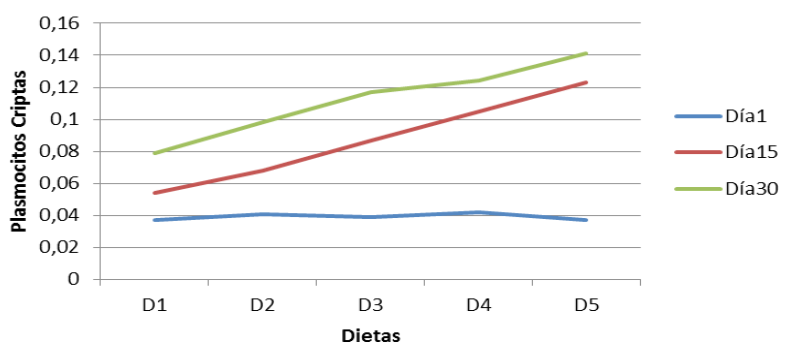

Figura 11. Plasmocitos en criptas de cerdos, con y sin adición de cepas probióticas (Plasmocytes in crypts of pig, with and without addition of probiotic strains).

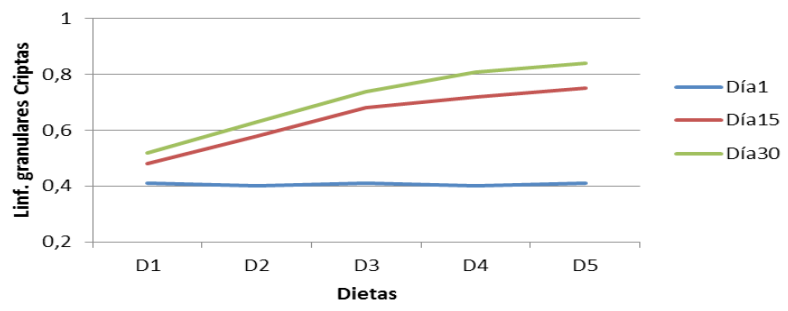

Figura 12. Linf. granulares Criptas de cerdos, con y sin adición de cepas probióticas (Granular Linf. in crypts of pigs with and without addition of probiotic strains).

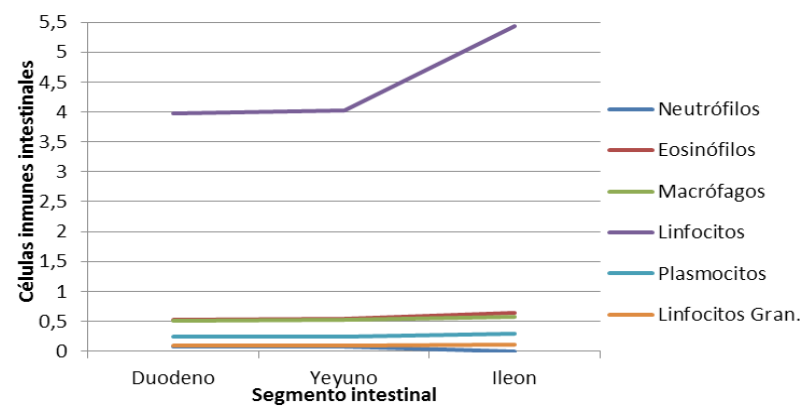

Figura 13. Células inmunes en vellosidades intestinales de cerdos, con y sin adición de cepas probióticas (Immune cells in villi of pigs with and without addition of probiotic strains).

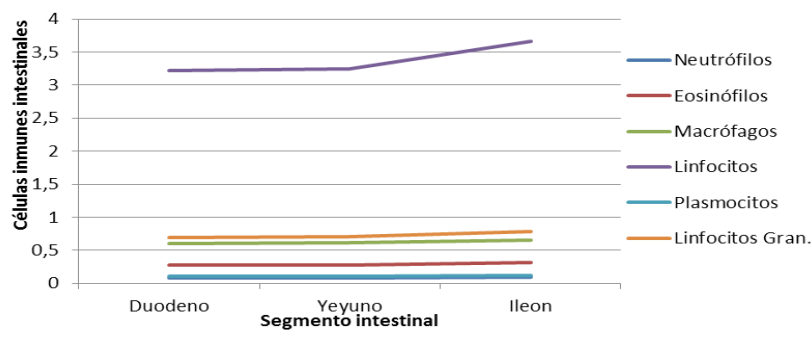

Figura 14. Células inmunes en criptas intestinales de cerdos con y sin adición de cepas probióticas (Immune cells in intestinal crypts pigs with and without addition of probiotic strains).

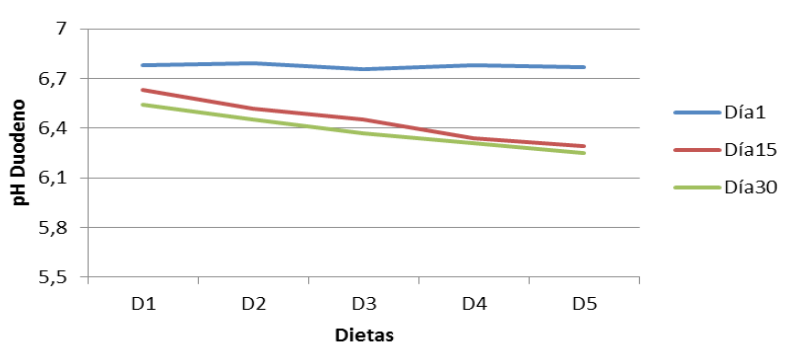

Figura 15. $\mathrm{pH}$ en duodeno de cerdos con y sin adición de cepas probióticas (Duodenal pH of pigs with and without addition of probiotic strains).

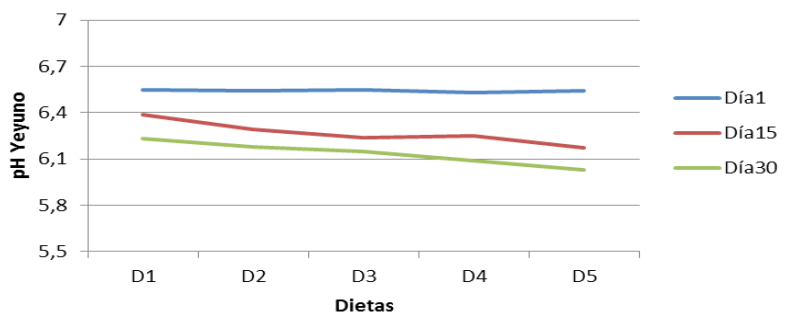

Figura 16. pH en yeyuno de cerdos con y sin adición de cepas probióticas (Jejunum $\mathrm{pH}$ of pigs with and without addition of probiotic strains). 


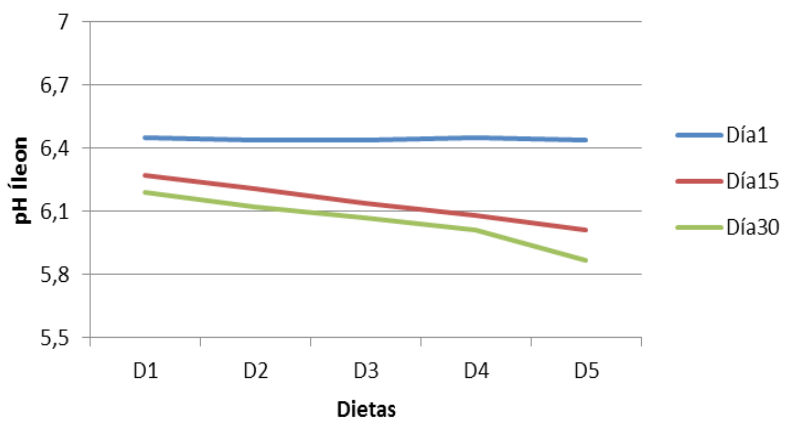

Figura 17. pH en Íleon de cerdos, con y sin adición de cepas probióticas (lleum pH of pigs, with and without addition of probiotic strains).

las células M. La administración oral de bacterias probióticas puede provocar algún tipo de interacción con la superficie de la mucosa, la cual puede ser traducida en una activación de las CEI e inducir la liberación de citoquinas antiproinflamatorias, ya que dicha interacción se realiza a través de receptores tipo Toll (TRL), evitando una respuesta inmune inflamatoria. Después de que las bacterias probióticas o sus fragmentos hacen contacto con las células dendríticas o los macrófagos en la lámina propia de la mucosa intestinal, son fagocitadas a través de los receptores de membrana, receptor TLR-2, y activan estas células. La activación produce la liberación de citoquinas reguladoras o proinflamatorias sin alteración de la homeostasis intestinal. Además, no se produce presentación antigénica, incluso cuando el probiótico interactúa con las células inmunes de las placas de Peyer (sitio inductor de inmunidad), ya que no se encuentran anticuerpos contra BAL (Galdeano y Perdigón, 2006). Como se puede observar en los resultados de nuestra investigación, se presentó una respuesta inmune controlada en el intestino delgado, evitando la hipersensibilidad que pudiese causar el probiótico como agente extraño.

Varios trabajos han demostrado que el reconocimiento microbiano por células epiteliales intestinales (CEI) es un aspecto integral de respuesta del hospedador de primera línea, apuntando a la idea de que el epitelio es más que simplemente una barrera física que separa contenido luminal de las células presentadoras de antígenos (CPA) de la mucosa (Villena et al., 2012; Villena et al., 2013). Es cada vez más reconocido que el epitelio intestinal está jugando un papel esencial en la homeostasis inmune, mediante la promoción de respuestas tolerogénicas y regulatorias. Estos resultados tienen implicaciones importantes sobre la regulación de la homeostasis de la mucosa por las bacterias probióticas (Suda et al., 2014), explicando la regulación de la respuesta inmune contra los probióticos. Como se observó en los resultados de esta investigación se presentó un proceso de tolerancia a favor de los probióticos y, por ende, una adaptación a dichos microorganismos. Esto se demuestra con la activación regulada del sistema inmune, mediante un aumento significativo de las poblaciones celulares inmunes en los lechones suplementados con cepas probióticas, en comparación con los lechones de los grupos control (antibiótico y sin adición de cepa probiótica ni antibiótico) a lo largo del experimento. Además, se pudo determinar un aumento significativo de las células inmunes intestinales en los lechones que fueron suplementados con E. faecium.

Por último, Franz et al. (2011) demostraron que la administración de probióticos condujo a una reducción significativa en el pH intestinal. Lo anterior coincide con los resultados de nuestra investigación, donde se observó una disminución significativa del $\mathrm{pH}$ intestinal, a lo largo del intestino y durante los diferentes días de evaluación. Estos resultados apoyan la hipótesis de que los probióticos pueden inhibir las cepas patógenas en el intestino a través del descenso del valor de $\mathrm{pH}$, ya que este $\mathrm{pH}$ crea un ambiente favorable para la proliferación de los probióticos, y esto a su vez, aumenta la exclusión competitiva por parte de las cepas probióticas hacia los microorganismos patógenos.

Por todo lo desarrollado anteriormente, los microorganismos probióticos no solo actúan a nivel intestinal estimulando la flora local, sino también, afectando otros órganos a través de estímulos inmunológicos, los cuales modulan la permeabilidad intestinal, la translocación bacteriana, y la producción de metabolitos bioactivos (Pala et al., 2011; Zhu et al., 2013).

\section{CONCLUSIÓN}

El destete está asociado con múltiples factores que generan la presentación de estrés en los animales, los cuales provocan un desequilibrio entre la tolerancia y la inmunidad en el intestino lo que puede contribuir a numerosas patogénesis de condiciones inflamatorias. Los resultados de la investigación indican que las cepas probióticas inducen el aumento en el número de poblaciones celulares inmunológicas intestinales. Además, los probióticos lograron un mayor descenso del valor de $\mathrm{pH}$, creando un ambiente favorable para su proliferación, y a su vez, aumentando la exclusión competitiva contra patógenos, siendo representativo en los lechones suplementados con la cepa probiótica E. faecium. Por lo anterior, las cepas de probióticos, específicamente E. faecium, inducen las diferentes redes inmunológicas ayudando a mantener la tolerancia intestinal y mejorando el desarrollo de respuestas inmunes protectoras y controladas.

\section{FINANCIACIÓN}

Proyecto financiado por la Universidad Nacional de Colombia, Sede Medellin (DIME).

\section{BIBLIOGRAFÍA}

Abraham, C. and Medzhitov, R. 2011. Interactions between the host innate immune system and microbes in inflammatory bowel disease. Gastroenterology, 140: 1729-1737.

Allen, H.K.; Levine, U.Y.; Looft, T.; Bandrick, M. and Casey, T.A. 2012. Treatment, promotion, commotion: antibiotic alternatives in foodproducing animals. Trends Microbiol, 21: 114-119.

Baver, E.; Williams, B.A.; Smidt, H.; Verstegen, M.W. and Mosenthin, R. 2006. Influence of the gastrointestinal microbiotia on development 
of the immune system in young animals. Curr Issues Intest Microbiol, 7: $35-51$.

Bermudez-Brito, M.; Plaza-Díaz, J.; Muñoz-Quezada, S.; GómezLlorente, C. and Gil A. 2012. Probiotic mechanisms of action. Ann Nutr Metab, 61: 160-174.

Boehm, M.; Hoy, B.; Rohde, M.; Tegtmeyer, N.; Bæk, K.T.; Oyarzabal, O.A.; Brøndsted, L.; Wessler, S. and Backert, S. 2012. Rapid paracellular transmigration of Campylobacter jejuniacross polarized epithelial cells without affecting TER: role of proteolytic-active HtrA cleaving E-cadherin but not fibronectin. Gut Pathog, 4: 3.

CIOMS (Council for International Organizations of Medical Sciences). 1985. International Guiding Principles for Biomedical Research Involving Animals. Geneva. 28 pp.

Deegan, L.H.; Cotter, P.D.; Hill, C. and Ross, P. 2006. Bacteriocins: Biological tools for biopreservation and shelf-life extension. Int Dairy J, 16: 1058-1071.

Franz, C.M.; Huch, M.; Abriouel, H.; Holzapfel, W. and Gálvezb, A. 2011. Enterococci as probiotics and their implications in food safety. Int J Food Microbiol, 151: 125-140.

Galdeano, C.M. and Perdigon, G. 2006. The probiotic bacterium Lactobacillus casei induces activation of the gut mucosal immune system through innate immunity. CVI, 13: 219-226.

Galdeano, C.M.; Dogi, C.A.; Bibas Bonet, M.E.; de Moreno de LeBlanc, A. and Perdigón, G. 2013. Chapter 18: Probiotic bacteria as mucosal immune system adjuvants. In: R.R. Watson and V.R. Preedy (Eds.). Bioactive food as dietary interventions for liver and gastrointestinal disease. Elsevier Inc. Argentina.

Ghadimi, D.; d Vrese, M.; Heller, K.J. and Schrezenmeir, J. 2010. Effect of natural commensal-origin DNA on toll-like receptor 9 (TLR9) signaling cascade, chemokine IL-8 expression, and barrier integritiy of polarized intestinal epithelial cells. Inflamm Bowel Dis, 16: 410-427.

Gómez-Llorente, C.; Muñoz, S. and Gil, A. 2010. Role of toll-like receptors in the development of immunotolerance mediated by probiotics. Proc Nutr Soc, 69: 381-389.

Gutiérrez, V.C.; Román, O.Y; Peláez, J.C.; Ciro, J.; López, H.A. y Parra, S.J. 2012. Efecto de la adición ex vivo del lipopolisacárido de Escherichia coli sobre la absorción de lisina en cerdos destete. Rev Fac Nal Agr Medellín, 65: 6447-6457.

Hansen, C.H.F.; Nielsen, D.S.; Kverka, M.; Zakostelska, Z.; Klimesova, K.; Hudcovic, T.; Tlaskalova-Hogenova, H. and Hansen, A.K. 2012. Patterns of early gut colonization shape future immune responses of the host. PLoS ONE, 7: e34043.

Heo, J.M.; Opapeju, F.O.; Pluske, J.R.; Kim, J.C.; Hampson, D.J. and Nyachoti, C.M. 2013. Gastrointestinal health and function in weaned pigs: a review of feeding strategies to control post-weaning diarrhoea without using in-feed antimicrobial compounds. J Anim Physiol Anim Nutr, 97: 207-237.

Jaramillo, A. 2011 . Evaluación de la mezcla de un prebiótico y un ácido orgánico en la salud intestinal y parámetros productivos de pollos de engorde. Rev Colombiana de Ciencia Animal, 5 (1): 52-66.

Jurado, G.H.; Ramírez, C. y Martínez, J. 2013. Evaluación in vivo de Lactobacillus plantarum como alternativa al uso de antibióticos en lechones. Rev MVZ Córdoba, 18: 3648-3657.

Martin, L.; Pieper, R; Kröger, S.; Goodarzi Boroojenia, F.; Vahjen, W.; Neumann, K.; Van Kessel, A.G. and Zentek, J. 2012. Influence of age and Enterococcus faecium NCIMB 10415 on development of small intestinal digestive physiology in piglets. Anim Feed Sci Tech, 175: 65-75.

Mejía-Medina, J.; Rincón-Ruiz, J.; Gutiérrez-Vergara, C.; Correa, G.; López-Herrera, A. y Parra-Suescún, J. 2012. Valoración de pará- metros clínicos y lesiones en órganos de cerdos durante el período posdestete. Acta Agronómica, 61: 61-68.

Montoya, R.C.; López, H.A. y Parra, S.J. 2012. Alteraciones en la producción mRNA de enzimas intestinales de cerdos durante varios períodos posdestete. Biotecnología en el Sector Agropecuario y Agroindustrial, 10: 126-134.

Nabuurs, G.J. and Mohren, G.M.J. 1993. Carbon fixation through forestation activities: a study of the carbon sequestering potential of selected forest types, commissioned by the Foundation Face. Institute for Forestry and Nature Research, Wageningen. IBN Research Report 93/4. 205 pp.

NRC. Nutrient Requirements Council. 2012. The nutrient requirements of swine. Eleventh Revised Edition. National Academy of Sciences. Washington, D.C.

Ospina, D.; Ciro, J; Román, Y.; Peláez, C.; López, A. y Parra, J. 2011. Cambios en la actividad enzimática en duodeno y yeyuno de cerdos durante varios periodos posdestete. Rev Med Vet Zoot, 58: 156-165.

Pala, V.; Sieri, S.; Berrino, F.; Vineis, P.; Sacerdote, C.; Palli, D.; Masala, G.; Panico, S.; Mattiello, A.; Tumino, R.; Giurdanella, M.C.; Agnoli, C.; Grioni, S. and Krogh, V. 2011 . Yogurt consumption and risk of colorectal cancer in the Italian European prospective investigation into cancer and nutrition cohort. Int J Cancer, 129: 2712-2719.

Reis, S.T.C.; Guerrero, C.M.J.; Aguilera, B.A. y Mariscal, L.G. 2005. Efecto de diferentes cereales sobre la morfología intestinal de lechones recién destetados. Téc Pecu Mex, 43: 309-321.

Saad, N.; Delattre, C.; Urdaci, M.; Schmitter, J.M. and Bressollier, P.M. 2013. An overview of the last advances in probiotic and prebiotic field. Food Sci Technol, 50: 1-16

SAS® Institute Inc. Statistical Analysis Systems Institute. 2007. SAS/ STAT User's Guide, Version 9.1 th Ed.: SAS Institute Inc. Cary, NC.

Segalés, J. y Domingo, M. 2003. La necropsia en el ganado porcino, diagnóstico anatomopatológico y toma de muestras.Boehringer Ingelheim. Madrid (España). pp. 10-14.

Suárez-Belloch, J. and Latorre, M.A. 2014. Nutrición y salud intestinal de los lechones al destete. IVIS SUIS, 105: 14-19.

Suda, Y.; Villena, J.; Takahashi, Y.; Hosoya, S.; Tomosada, Y.; Tsukida, K.; Shimazu, T.; Aso, H.; Tohno, M.; Ishida, M.; Makino, S.; Ikegami, S. and Kitazawa, H. 2014. Immunobiotic Lactobacillus jensenii as immune-health promoting factor to improve growth performance and productivity in post-weaning pigs. BMC Immunology, 15: 24.

Villena, J. and Kitazaw, H. 2013. Chapter 5: Role of toll-like receptors in the modulation of intestinal inflammation by immunobiotics. In: Kitazawa H.; Villena J. and Alvarez A. (Eds.). Probiotics: Immunobiotics and immunogenics. CRC Press. Boca Raton, FL. USA. pp. 89-127.

Villena, J.; Aso, H.; Alvarez, S. and Kitazawa, H. 2012. Chapter 2: Porcine toll-like receptors and their crosstalk with immunobiotics: impact in the regulation of gut inflammatory immunity.In: Smith, A.; Jones, C.J. (Eds.). Probiotics: Sources, types and health benefits. Nova Science Publishers. New York. USA. pp. 53-84.

Walker, W.A.; Sanderson, D.J.; Churcher, C.; Parkes, C.G.; Hudspith, N.B.; Rayment, N.; Brostoff, J.; Parkhill, J.; Dougan, G. and Petrovska, L. 2012. High-throughput clone library analysis of the mucosaassociated microbiota reveals dysbiosis and differences between inflamed and noninflamed regions of the intestine in inflammatory bowel disease. BMC Microbiology, 11: 7.

Zacharofa, M.P. and Lovittb, R.W. 2012. Bacteriocins produced by lactic acid bacteria. A review article. APCBEE Procedia, 2: 50 - 56.

Zhu, Q.; Gao, R.; Wu, W. and Qin, H. 2013. The role of gut microbiota in the pathogenesis of colorectal cancer. Tumor Biology, 34: 1285-1300. 